\title{
Survey Paper on Web Recommendation System
}

\author{
Megha Sen ${ }^{1}$, Seema Udgirikar ${ }^{2}$ \\ ${ }^{1}$ Savitribai Phule Pune University, GH Raisoni College of Engineering and Management, Pune, India \\ ${ }^{2}$ GH Raisoni college of Engineering and Management, Savitribai Phule Pune University, Pune, India
}

\begin{abstract}
The internet has become a significant source of information .it has provide platform for many e-commerce enterprises. These e-commerce sites have very broad variety of products and have loads of information about the product or services, so it is difficult for the customers to choose best product according to their needs. To overcome the problem of information overload, many Recommendation techniques have been proposed earlier. A personalized recommendation system can handle this issue. There are still challenges in Recommender system 1)The items and user profiles in e-commerce sites such as e-learning, e-business are so complex and vague so they can be described as complex tree structure. 2) Attributes of items and user behavior are subjective, vague and imprecise. These in turn induce uncertainty in representing and reasoning on items' features, users' behavior, and their relationships so fuzzy set theory is used to handle this uncertainty. An item tree and user request tree-based hybrid recommendation approach is then developed. To model users' fuzzy tree-structured preferences, a fuzzy preference tree model is proposed. A fuzzy preference tree-based recommendation approach is then developed. Experimental results on an Australian business dataset and the Movie lens dataset show that the proposed recommendation approach have good performance and handled tree-structured data efficiently.
\end{abstract}

Keywords: E-business, fuzzy preferences, recommender systems, tree matching, web-based support system

\section{Introduction}

E-commerce web-sites are providing new business portals and large amount of product information, so customers spend more and more time browsing the net in order to find the right information or product. One solution to overcome with this problem is to develop a personalized Recommendation system. This system retrieves the information desired by the customer and helps him in determining which product to buy.

In CF systems user ratings are expressed as binary values. Rating greater than 3 considered as item liked by the user and less than 3 as item, disliked by the user. Same rating can have different meaning to different users so ratings depend on the particular human thinking process. Same rating say 3 on scale of 5 does not mean equal degree of interest in an item. This all contributes to Fuzziness.

Item attributes and user behavior are subjective, not clear and inaccurate. These all contributes to uncertainty. How to represent uncertainty of item features and user behavior? To handle this uncertainty Fuzzy theory is used to represent item features and user behavior. Fuzzy theory represents item and user preferences as vector accordingly.

Items or user profiles in a B2B environment have complicated structures, such as tree structures. So recommendation systems have hardly used in the B2B environment. For example, a business in a B2B application environment, there are many product categories, each of

This may have many of subcategories, under which there may be multiple specific products, which together form a tree structure. In earlier approaches, an item is normally described as a single value or a vector. The fuzzy preference models cannot deal with tree-structured data in a Web-based B2B environment.
This study resolve challenges like, Tree structured data, tree structured user preferences, uncertainty in user preferences, and personalization problem in Recommendation system and propose a fuzzy tree structured user preference tree based recommendation system.

\section{Literature Survey}

Recommendation System Techniques

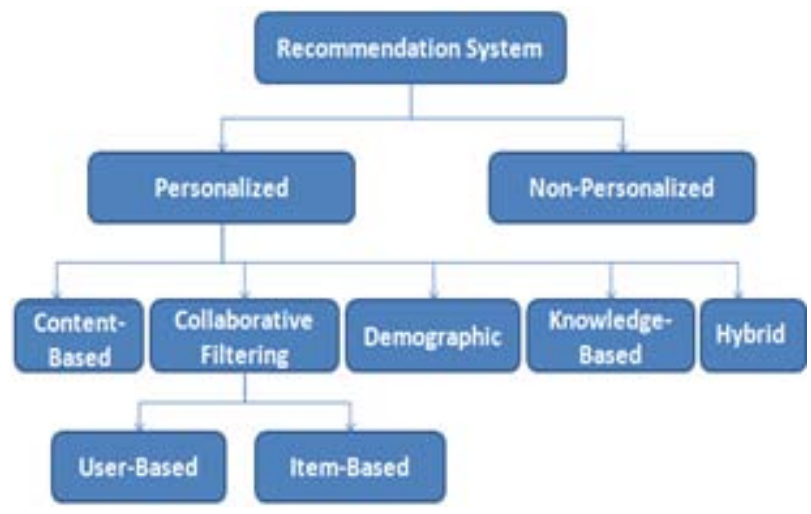

1)Collaborative Filtering: A collaborative filtering recommendation system recommend items to the user based upon the recommendation of similar user's. similarity of users are find out using past history(ratings) of user,s.if two users liked an item in the past then they would like an item in the future also.

2)Content based Recommender Systems: Content based recommendation system recommend items to the user based on the items purchased in the past history and profile of the user then user profiles are compared with the new items .items which matches with the user profile are recommended to the user.

3)Knowledge base Recommendation system: There are many items which are not purchased frequently so very less ratings are available for these items. In this case knowledge based recommendation system is used. User gives his

\section{Volume 4 Issue 11, November 2015}




\section{International Journal of Science and Research (IJSR) \\ ISSN (Online): 2319-7064}

Index Copernicus Value (2013): 6.14 | Impact Factor (2014): 5.611

preferences for the items then items are recommended based on these preferences.

4)Demographic: This technique use information about the user like age, gender, location, occupation etc. this demographic information is used to find out similar users. Items are recommended based on ratings of similar users.

5)Hybrid Recommendation system: this technique combined any two approaches to remove issues in recommendation technique.

\section{Issues in Recommendation System}

1)Data Sparsity: When there is very less ratings about items then it is very difficult to recommend items to the user.CF systems suffer from this problem.

2)Cold Start: There are two kinds of cold start problem, new user and new item problem. There is no information about new user and new item so it is difficult to recommend items.

3) Scalability: It is the ability of recommendation system to handle growing amount of information. Information about the user and item grows rapidly on the internet. CF systems Becomes expensive to handle growing amount of information and gives inaccurate recommendations.

4)Over Specialization: Recommendation systems recommend items based on previous history. User's does not get diversified recommendations.

[1]. this paper proposes a Tag based collaborative filtering Recommendation approach for personal learning Environments (PLE), s. Here 16 different tags based collaborative Filtering recommendation algorithms are implemented and compared in terms of accuracy and user satisfaction. PLE learners have Different interest and preferences so there is no similarity in their learning resources. User generated tags are combined with traditional collaborative filtering recommendation. <User-item $>$ Relation converted into the $<$ user, item, tag $>$ relation. The result shows that item based K-means clustering Algorithm gives best performance in offline evaluation. Whereas in the user evaluation user based Apriori Algorithm ranked First. The result of evaluation shows that there is no relation between quality of user experience and high recommendation accuracy measured by statistical measure.

[2].This paper proposes a personalized Recommendation approach based on Three Social Factors ,Personal interest means user-item relationship and interpersonal influence and interpersonal interest similarity means user-user relationship of social networks.probablistic matrix Factorization is used Experiments are done on three datasets Movielens and yelp. This approach removes the problem of cold start and data sparsity.

[3].This paper proposes a recommendation system for real estate websites that helps users in purchasing new properties or homes. Recommendation system is developed by combining case based reasoning (CBR) and Ontology. Earlier systems supports single attribute search systems but this system support multivalued search system. User search behaviors are studied and a knowledge base is prepared.
Then the semantic meaning of attributes and relationship between them is defined by ontology. Result shows that this approach is efficient and affordable for housing search in real estate websites.

[4] This paper proposes a hybrid collaborative filtering recommendation approach based on user preferences and item features. Traditional collaborative filtering recommendation approach has challenges like 1). Data sparsity 2).scalability 3). Similarity-: similar items can have different names and meaning so recommendation system would treat them as different items. To solve these challenges a recommendation algorithm is proposed based on user preferences and item -features. User preferences are determined from previous ratings of user over a period of time and then using similarity matrix find similar users. An item is additional represented as vector of different features. Every feature has given weight. If a feature is visible in different items then it becomes the preference of user. The proposed algorithm is more accurate than other traditional CF methods. it also removes the problem of data sparsity to some extent.

[5].This paper proposes a new approach of content based Recommendation system that is based on Transfer learning. This approach solves the problem of data sparsity when there is lack of information in target domain but there is sufficient information in other domain. A behavior graph model is prepared .From the user preferences this graph is then converted into the tree called Behavior tree. For each tree in source domain a corresponding tree in target domain is find out. BGM method is compared with other cross domain methods like KNN cross domain method. The result shows that performance of $\mathrm{BGM}$ is better than $\mathrm{KNN}$.

[6].A hybrid recommendation approach for e- learning environment is proposed. Two types of attributes are considered for learning resources 1.Explicit attributes like subject and name of the publisher 2.Implicit attributes can be extracted from the historical ratings of learners. Explicit attribute based RS and implicit attribute based RS prepared and combined to give accurate recommendation, $\mathrm{s}$ for learners. In EAB-RS ,a Learner preference tree (LPT) is prepared for each learner from historical logs and ratings then similarity between learners is find out and rating prediction for resources is given by NNCF Algorithm. In IAB-RS ,implicit attributes are extracted using Genetic algorithms $(\mathrm{GA})$ then similar users are find out and finally rating prediction is done by NNCF. This recommendation approach resolves the problem of Data sparsity, cold start and provide more diverse recommendation list. 


\section{International Journal of Science and Research (IJSR) \\ ISSN (Online): 2319-7064}

Index Copernicus Value (2013): 6.14 | Impact Factor (2014): 5.611

\begin{tabular}{|c|c|c|c|}
\hline \multicolumn{4}{|c|}{ Literature Survey Table } \\
\hline Paper & Technique & Algorithm used & pros and cons \\
\hline$[1]$ & CF based & $\begin{array}{c}\text { K-means clustering, } \\
\text { Apriori, } \\
\text { NBC, } \\
\text { KNN, etc. }\end{array}$ & $\begin{array}{l}\text { Result shows that user } \\
\text { experience does not relate } \\
\text { with high recommendation } \\
\text { accuracy. }\end{array}$ \\
\hline$[2]$ & CF based & $\begin{array}{c}\text { Probabilistic-tic } \\
\text { matrix factorization- } \\
\text { on }\end{array}$ & $\begin{array}{l}\text { Removes the problem of } \\
\text { cold start and data sparsity }\end{array}$ \\
\hline$[3]$ & $\begin{array}{c}\text { Knowledge } \\
\text { based }\end{array}$ & $\begin{array}{l}\text { Case based } \\
\text { reasoning and } \\
\text { ontology }\end{array}$ & $\begin{array}{l}\text { Gives a multiattribute } \\
\text { search system }\end{array}$ \\
\hline$[4]$ & $\begin{array}{c}\text { Hybrid CF } \\
\text { method }\end{array}$ & TF-IDF model & $\begin{array}{l}\text { 1.More accurate than other } \\
\text { CF methods } \\
\text { 2.removes the problem of } \\
\text { data sparsity to some } \\
\text { extent }\end{array}$ \\
\hline$[5]$ & $\begin{array}{c}\text { Content } \\
\text { based }\end{array}$ & BGM method & $\begin{array}{l}\text { Solve the problem of data } \\
\text { sparsity }\end{array}$ \\
\hline$[6]$ & $\begin{array}{c}\text { Hybrid } \\
\text { Approach }\end{array}$ & Nearest neighbor $\mathrm{CF}$ & $\begin{array}{l}\text { 1. Remove cold start and } \\
\text { data sparsity issue } \\
\text { 2. Gives diversified results }\end{array}$ \\
\hline
\end{tabular}

This recommendation approach solve the

1) Cold start issue more efficiently than other approaches because new user preferences are added with the previous preferences.

2) Data sparsity issue because user-item matrix is not considered here for similarity.

3) Scalability issue because user fuzzy preference tree updated efficiently.

4) In future we will work on developing methods for group recommendations.

\section{References}

[1] Mohamed Amine Chatti, Simona Dakova, Hendrik Thu s, and Ulrik Schroeder, "Tag-Based Collaborative Filtering Recommendation in Personal Learning Environments", VOL. 6, NO. 4, OCTOBERDECEMBER 2013

[2] Liang Hu, Guohang Song, Zhenzhen Xie, and Kuo Zhao, "Personalized Recommendation Algorithm Based on Preference Features", TSINGHUA SCIENCE AND TECHNOLOGY ISSN 1007-0214 08/11 pp293-299 Volume 19, Number 3, June 2014

Existing Recommendation system handles the tree structured data. User preferences are fuzzy means they cannot be expressed by a number so fuzzy set theory is used to describe complex preferences of users. User preferences are collected by two ways.

1)Intentionally Expressed preferences: This is directly given by the user. Items have different features that forms the tree structure.so user preferences are in tree structures. User assigns a preference value to each feature which is expressed in terms of fuzzy set. Suppose expressed as T1.

2)Extensionally Expressed preferences: These preferences are constructed from the items previously purchased or experienced by the user. Each item represents a tree associated with a preference value given by the user. Suppose expressed as T2. The users Fuzzy preference based tree is constructed by combining T1 and T2. There are two steps for combining the two trees.

a) There is a need to find the parts of tree are which matches conceptually. For this a conceptual similarity Tree mapping algorithm is used.

b)Fuzzy preference tree matching algorithm is used to construct Fuzzy preference tree of user.

Fuzzy preference Recommendation Approach:

This system takes two inputs

a) Maximum conceptual tree similarity mapping between two trees $\mathrm{T} 1$ and $\mathrm{T} 2$.

b) Fuzzy preference tree node

This system calculates prediction rating of user $\mathrm{u}$ for item I by prediction rating algorithm.

\section{Conclusion}

This paper proposes a recommendation approach for e business or e-commerce websites that have very complex product/services categories. User's fuzzy preference tree is compared with the target item tree and predicted rating of the item is calculated by this approach. 